\title{
A global assessment of climatic niche shifts and human influence in insect invasions
}

\author{
Matthew P. Hill ${ }^{1}$ () | Belinda Gallardo ${ }^{2}$ | John S. Terblanche ${ }^{1}$
}

${ }^{1}$ Centre for Invasion Biology, Department of Conservation Ecology and Entomology, Faculty of Agrisciences, Stellenbosch University, Private Bag X1, Matieland, 7602, South Africa

${ }^{2}$ IPE-CSIC Pyrenean Institute of Ecology, Department of Biodiversity Conservation and Ecosystem Restoration, Applied and Restoration Ecology Group, Avda. Montañana 1005, Zaragoza, 50059, Spain

\section{Correspondence}

Matt Hill, Centre for Invasion Biology, Department of Conservation Ecology and Entomology, Faculty of Agrisciences, Stellenbosch University, Private Bag X1, Matieland, 7602, South Africa.

Email: matthill@protonmail.com

Editor: Dr. Petr Keil

\begin{abstract}
Aim: Non-native invasive insects have major impacts on ecosystem function, agricultural production and human health. To predict the geographical distributions of these species, correlative ecological niche models (ENMs) are typically used. Such methods rely on assumptions of niche conservatism, although there is increasing evidence that many species undergo niche shifts during invasions. The magnitude and direction of niche shifts, however, is likely to vary within and between taxonomic groups, highlighting that an assessment of potential niche shifts in such insects is required.
\end{abstract}

Location: Global.

Time period: Current.

Major taxa studied: Insects.

Methods: We compile a novel database of 22 globally invasive, non-native insect species and test for niche expansion and unfilling across this group. We examine if factors such as the native range size, poleward shifts and human influence may be associated with observed niche changes. Finally, we construct ENMs and examine the reliability of their predictions in light of our niche shift results.

Results: Niche expansion was apparent in 12 of the 22 species, suggesting that altered speciesclimate relationships during invasion is common for this group. Likewise, niche unfilling occurred in 15 of the species. Increasing human disturbance (combining human population, transport networks and land use) explained $40 \%$ of observed niche expansions and $54 \%$ of incidents of niche unfilling. Niche metrics and ENM performance were sensitive to the choice of background extents.

Main conclusions: Many non-native insects expand into new climates in their invasive ranges. The prevalence of niche unfilling across this group suggests climate disequilibrium and the potential for further range expansion. Non-native invasive insects tend to invade areas with similar human disturbance to their native range, and habitat accessibility appears important for these species to achieve their full invasion range potential. Ideally, ENMs should not be used in isolation for this group, but should be coupled with other methods or experiments to test for potential niche change.

KEYWORDS

ecological niche modelling, human disturbance, insects, invasive species, niche shift

\section{1 | INTRODUCTION}

The introduction and spread of non-native invasive species constitutes one of the most important drivers of global change, triggering impacts that extend well beyond the loss of biodiversity and disruption of ecosystem services, and affect human well-being in various ways (Vitousek, D'Antonio, Loope, \& Westbrooks, 1996). Of the different types of invaders, insects comprise some of the most recognized vectors of human disease (Lounibos, 2002) and cause billions of dollars in direct agricultural losses through reduced yields and transmission of 
plant pathogens, and indirect losses including increased application of pesticides and trade restrictions (Bradshaw et al., 2016). Predicting the distribution, spread and impacts of non-native invasive insects is thus an important challenge (Hill, Clusella-Trullas, Terblanche, \& Richardson, 2016) and is required to inform spatial prioritization and policies in order to prevent new invasions and manage existing invasive species (Gallardo \& Aldridge, 2013).

In attempts to understand how species may respond to novel environments, ecologists typically employ some concept of the niche to characterize the limits to species geographical distributions. Whilst characterizing the fundamental niche (i.e., the full range of physical and biological requirements for a species; Wiens, Stralberg, Jongsomjit, Howell, \& Snyder, 2009) allows for more accurate predictions of distribution limits, extensive ecophysiological data are required. However, the available data for many invasive species are typically limited to observations, or geographical presence data. For this reason, ecological niche models (ENMs) have become increasingly popular for predicting the potential geographical range of non-native invasive species. Such models correlate the known distribution of a species with environmental variables, typically climate, across known localities. By characterizing species-environment relationships in this way it is possible to approximate the realized niche (Guisan, Petitpierre, Broennimann, Daehler, \& Kueffer, 2014), that is the proportion of the fundamental niche actually occupied by the species, due to limits set by both biotic and abiotic interactions (Wiens et al., 2009) and dispersal limitations. This use of realized niches and climatic variables is close to the Grinnellian definition of the niche (Wiens et al., 2009), and is useful for describing distribution patterns at broad scales of invasion (Broennimann et al., 2012).

The ability of ENMs to accurately predict non-native ranges relies on adequately sampled distribution data, and inherently makes assumptions of climate equilibrium and niche conservatism (Mateo et al., 2015). Species that occur in all available suitable climates are said to be in equilibrium with climate (Petitpierre et al., 2012; Wiens et al., 2009). Equilibrium for non-native invasive species particularly relies on opportunities to reach these climates, and may reflect the stage of the invasion process for the species. Related to this is the idea of niche conservatism, which describes species that establish in novel geographical regions that hold similar climatic space to the range in which they evolved (Broennimann et al., 2007; Wiens \& Graham, 2005). Conversely, deviations in species-environment relationships that result in expansion and persistence of non-native invasive species in areas that were not predictable from quantifying the native range alone are termed niche shifts. Niche shifts are of particular importance for predicting distributions and impacts of non-native species as they may be facilitated through a range of processes, including release from competition and predation, overcoming dispersal limitations, human transformation of habitats or through adaptive shifts in niche-limiting traits (Hill, Chown, \& Hoffmann, 2013; Guisan et al., 2014). Across different taxa there are increasing examples of niche shifts during invasion; these examples challenge the assumption of niche conservatism and bring the validity of applying ENM techniques to different invasive species into question ( $\mathrm{Li}$, Liu, $\mathrm{Li}$, Petitpierre, \& Guisan, 2014; Parravicini, Azzurro, Kulbicki, \& Belmaker,
2015), especially for those groups showing high amounts of niche expansion (Early \& Sax, 2014; Li et al., 2014; Parravicini et al., 2015).

A recent proposal to unify studies of species niche shift measured in multivariate environmental space (Guisan et al., 2014) suggested important distinctions between measuring the level of true shifts, defined as niche expansions into climate space that was accessible in the native range but not occupied, and measuring niche unfilling, the portion of the climatic niche which is occupied in the native range but not yet exploited in the invaded or novel range (Broennimann et al., 2007). Niche unfilling measured in this way can also give an indication of non-equilibrium with climate in the novel range, perhaps due to dispersal constraints or limited opportunity to invade, and the potential of the species for further spread (González-Moreno, Diez, Richardson, \& Vilà, 2015). A number of recent studies have sought to investigate patterns of niche shift using these definitions across different groups, with the results being study- or context-specific. For example, Petitpierre et al. (2012) found low levels of niche expansion (i.e., high levels of niche conservatism) for terrestrial plant invaders, but Early \& Sax (2014) found high levels of niche expansion across (woody) plant invaders. Within vertebrate invasions, birds have displayed relatively low levels of niche expansion (Strubbe, Broennimann, Chiron, \& Matthysen, 2013), though among reptiles and amphibians (herpetofauna) niche expansion in the introduced environment was high (Li et al., 2014). Additionally, Strubbe, Beauchard, \& Matthysen (2015) found niche expansion was rare when looking at nonnative mammals, birds and amphibians in Europe.

While patterns of niche conservatism across non-native invasive insects are largely unknown, niche shifts have been reported for a number of insect species (e.g., da Mata, Tidon, Côrtes, De Marco, \& Diniz-Filho, 2010; Petersen, 2013). As many insect species are able to respond quickly to novel biotic interactions and environments (including environments impacted by humans; e.g., Hufbauer et al., 2012), through either phenotypic plasticity, adaptive trait changes or some combination of both (Gibert et al., 2016; Urbanski et al., 2012), niche shifts may be relatively common within this group. Identifying signals of niche shift can also lead to hypotheses surrounding potential drivers and mechanisms (e.g., adaptation in climate stress traits; Hill et al., 2013; Rey et al., 2012).

In this study, we collate distribution data for globally invasive, nonnative insect species to investigate patterns of niche shift and ENM transferability across this group. First, we determine the magnitude and types of niche shift across this group. Secondly, we test if factors that have been linked to niche change in other groups (e.g., plants, birds and herpetofauna), such as native range size, poleward expansions or human influence, are associated with niche shift in this assemblage of nonnative invasive insects. Finally, in light of the results, we discuss generally the reliability of ENMs for predicting distributions of invasive insects.

\section{2 | METHODS}

\subsection{Selection of species}

We compiled distribution data for non-native invasive insect species from the literature, databases and researchers, targeting those that 
have had recent attempts at ENM (see Supplementary Material 1 in the Supporting Information for a complete list of sources and numbers of observations). Data were presence-only because reliable absences for insect species are typically difficult to obtain; likewise we had little information on the dates of data collection. For a species to be included in our dataset it had to meet a set of criteria including: that it was globally invasive (transcontinental movement, as opposed to simply expansion into new environments that are adjacent to the native range), that it had a clear taxonomic identity (to avoid cryptic species); and it was of known geographical origin (see Supplementary Material 1). After applying these criteria, distribution data for 21 insect species and 1 mite species (Halotydeus destructor) were included (henceforth referred to as 'insects' for simplicity), with most of these data from the following taxonomic groups: (a) ants (Hymenoptera), (b) Coleoptera and (c) Diptera (Table 1, Supplementary Material 1). The mean number of data points for native and invasive distributions was highly variable: 115.7 ( \pm 96.3 ) and 408 ( \pm 580.5) points, respectively.

\section{2 | Defining ranges}

For many non-native invasive insects it is often difficult to determine the exact native range, as a species may also be considered invasive in areas near to their (original) native range, with such expansion often facilitated through adaptation to human-altered habitat (e.g., Leptinotarsa decemlineata and Wasmannia auropunctata; see Hufbauer et al., 2012). Consequently, we broadened the definition of native range to include nearby occurrence points to which the species may have expanded, so that our analysis was focused on measuring niche conservatism between disjoint geographical regions (see Supplementary Material 1). The invasive range was all the remaining points that fell outside this definition of native range.

\section{3 | Geographical background}

Geographical background refers to the area encompassing the native or invasive range plus the space accessible to the species which may not be colonized, for a number of reasons. Within these backgrounds is the range of climates available to the species. The way in which backgrounds are defined is likely to influence both ENM performance and niche change metrics (see below). For instance, larger geographical backgrounds (e.g., biogeographical realms) may be more favourable for increased transferability of ENMs for some invasive species, as well as being better suited to investigating niche conservatism across global scales of invasion (Mateo et al., 2015). The trade-off with large backgrounds is that ENMs may overfit environmental conditions (Anderson \& Raza, 2010) and yield increased amounts of niche unfilling (Mateo et al., 2015).

To explore some of the sensitivity of ENM performance and niche change metrics to background selection, we constructed three different geographical backgrounds. First, we selected all the ecoregions (using the definition of Olson et al., 2001) that held an occurrence point for both native and invasive ranges. Secondly, we selected ecoregions that held an occurrence point plus all ecoregions that were within the mean distance of ecoregion size (calculated radially) from each occurrence point (and fell within the same biomes, using the definition of Olson et al., 2001; see Supplementary Material 1). This second background allowed us to have much broader backgrounds than the first, but avoided overlap problems that occurred when selecting entire biomes. Finally, for the third background, we selected the unique Köppen-Geiger climate zones (Rubel \& Kottek, 2010) that held an occurrence point to provide an alternative selection procedure that has been used for non-native invasive insect ENMs elsewhere (e.g., Hill \& Terblanche, 2014) (Supplementary Material 2).

\section{4 | Climatic predictors}

It is necessary to employ the same climatic predictor set across all species to examine general patterns of niche conservatism across any taxonomic group (Guisan et al., 2014; Strubbe et al., 2015). Variables that are chosen to be biologically relevant (e.g., the physiological traits of climate stress resistance and life-history variation) to the species may produce better models (Rödder \& Lötters, 2009). We chose a set of eight climatic variables that are known to represent environmental dimensions that limit the distribution of terrestrial invertebrates and have been applied to a range of species, particularly dipterans (e.g., De Meyer et al., 2010; Hill \& Terblanche, 2014): mean diurnal temperature range, temperature seasonality, maximum temperature of the warmest month, minimum temperature of the coldest month, temperature annual range, precipitation of the wettest month, precipitation of the driest month and precipitation seasonality (Busby, 1991). These variables were derived from the University of East Anglia Climate Research Unit time series (CRU TS v.3.21) dataset (Harris, Jones, Osborn, \& Lister, 2014). Presence data were then rescaled to the predictor variable resolution of 10 arcmin (roughly $20 \mathrm{~km} \times 20 \mathrm{~km}$ at the equator) so that occurrences were limited to one per grid cell. For each geographical background we selected 10,000 random cells that did not hold a species presence record (or all available cells if fewer than 10,000 were available). We added these data to the species presence data and then extracted information for the eight climatic variables across all of these points to create presence/pseudo-absence datasets for each species.

\section{5 | Measuring niche shift}

Using the presence/pseudo-absence datasets, we conducted principal components analysis (PCA) (package 'ade4'; Dray \& Dufour, 2007) across the combined native and invasive ranges for each of the geographical backgrounds. Then, using the R package 'ecospat' (Broennimann et al., 2014) we took the first two PCA axes and rescaled them to a resolution of $100 \times 100$ grid cells (Broennimann et al., 2012; Petitpierre et al., 2012). The density of species occurrence points for each range was calculated using kernel smoothing methods (using the function 'ecospat::ecospat.grid.clim.dyn'; see Broennimann et al., 2012, 2014 for details) and then projected onto the rescaled PCA surface to create two-dimensional surfaces for native and invasive ranges. This rescaling and smoothing process allows for different ranges to be compared directly, as it reduces effects of sampling bias and missing data, 
TABLE 1 The 22 species used in this study with their associated niche metrics and environmental niche model performance

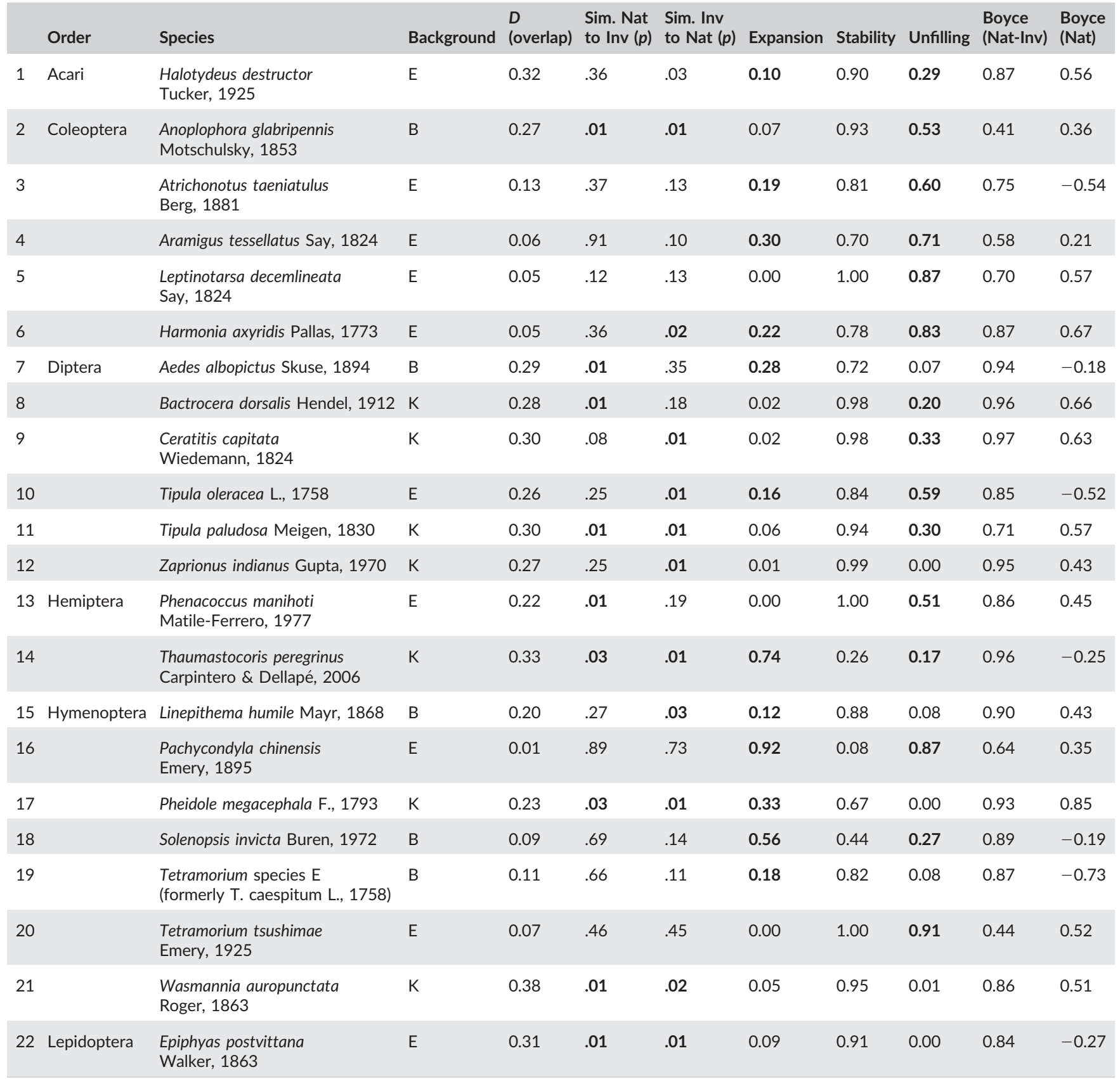

Niche overlap test (Schoener's D) in multivariate environmental space for the 22 non-native invasive insect species used in this study. Backgrounds are catergorised as either Ecoregion (E), Biome (B) or Koppen Geiger (K). Associated $p$ values for the niche similarity tests between the native compared to the invaded range (Sim. Nat to Inv (p)) and from invaded compared with native range (Sim. Inv to Nat (p)). Bold indicates significant values $(p<0.05)$ in these columns. Niche shift metrics include 'Expansion', 'Stability' and 'Unfilling'. Values range from 0 to 1 , with 1 being total niche expansion or unfilling and 0 reflecting no change between ranges. Bold values in these columns indicate that the amount of expansion or unfilling was above $10 \%$. Niche stability is 1-expansion. The Boyce index (Boyce Nat) indicates the performance of the native range data models alone in predicting the invasive range occurrences, with values ranging from 21 to 1 . The Boyce index (Boyce Nat-Inv) indicates the performance of the models combining native and invasive range data to predict the invasive range occurrences, with values ranging from 21 to 1 . Positive values indicate good model performance, values near 0 indicate models no better than random and negative values indicate predicting poor suitability where there are presences.

maximizes environmental differences between ranges, and allows for any differences in range size to be largely discounted (Broennimann et al., 2012; Li et al., 2014).

Using these two-dimensional surfaces, niche overlap was calculated using Schoener's $D$, which is a measure of similarity between the two surfaces and ranges from 0 (no overlap) to 1 (identical niches). We then conducted niche similarity tests (Warren, Glor, \& Turelli, 2008), as outlined in Broennimann et al. (2012), by randomly shifting the density of occurrences in one range and comparing overlap (Schoener's $D$ ) with the observed niche from the other range. This was repeated 100 times and then, using the observed $D$ score in a one-sided test, we examined whether the similarity of the native and invasive ranges was higher 
than expected by chance alone. We performed this test for both observed native ranges compared with randomized invasive ranges, and observed invasive ranges compared with randomized native ranges to provide two tests of niche similarity (Broennimann et al., 2012). In cases where the test was significant, we rejected that the niches were more similar than expected at random.

While this use of Schoener's $D$ can help to describe niche conservatism it does not account for distinctions within niche shifts. To investigate further, the niche shift metrics (a) niche expansion, (b) stability and (c) unfilling were calculated following Broennimann et al. (2012), Guisan et al. (2014) and Petitpierre et al. (2012) for all three different background extents. The native and invasive surfaces were overlaid and the niche metrics calculated at the intersection of the two occurrence densities within the total climate space available, to help distinguish between true niche shift and shifts due to climates not available in the native range. Niche expansion therefore describes climates that are available in both native and invasive ranges but only occupied in the invasive range (Guisan et al., 2014). Niche stability refers to those climates that are occupied in both native and invaded ranges. Niche unfilling describes climates that are available, but not yet exploited, in the invaded range, to give some indication of the degree of climate equilibrium. Values for expansion, stability and unfilling range from $0 \%$ to $100 \%$ and are typically said to be relevant at $>10 \%$, and although this threshold is partly arbitrary it allows for comparisons with other studies (e.g. Parravicini et al., 2015; Petitpierre et al., 2012; Strubbe et al., 2015). We calculated the niche shift metrics at the 75th percentile of density overlap between the two surfaces to reduce heterogeneity in climate availability between ranges. We also calculated the niche shift scores at the 80th, 85th, 90th, 95th and 100th percentiles to assess whether removal of marginal climates had any effect on these values (Guisan et al., 2014).

\section{6 | Factors associated with niche change}

We investigated whether native range size (measured through number of grid cells occupied) (Early \& Sax, 2014; Li et al., 2014) and the equatorial and poleward expansion in range shifts (expressed as a change in extremes from the native range in degrees latitude) (Li et al., 2014), may be associated with climatic niche shifts. Human disturbance may also facilitate invasions by increasing propagule pressure in areas far from the native range which the species could not access on its own, further promoting establishment (Gallardo, Zieritz, \& Aldridge, 2015), and has been associated with niche expansion (Strubbe et al., 2015). We included three variables related to human disturbance that have been shown to shape the global-scale distribution of invasive species (Gallardo et al., 2015): the Human Influence Index (HII), human population density and distance to roads. The HII (Sanderson et al., 2002) combines several factors presumed to exert an influence on ecosystems, such as human population distribution, urban areas, roads, navigable rivers and various agricultural land uses. The human population density (Oak Ridge National Laboratory, http://www.ornl.gov/sci/ landscan) has been shown to affect the broad-scale distribution of invasive species (e.g., Pyšek et al., 2010) and was thus included sepa- rately. Distance to roads, an indicator of accessibility and likely to be a proxy of propagule pressure, was calculated using the inverse of the Euclidean distance (in $\mathrm{km}$ ) from the occurrence of each species to the closest road, as described in Gallardo et al. (2015). All human variables were at the same global extent and resolution as other predictors used in this study (10 arcmin). Human disturbance values were extracted for each point occurrence and summarized separately for the native and invaded ranges of each species.

To investigate potential drivers of niche changes in globally invasive insects we calibrated regression models with the percentage of expansion and unfilling as the dependent variables and the different geographical (native range size, equatorial and poleward movement) and disturbance ( $\mathrm{HII}$, human population and distance to roads in the native and invaded ranges) factors as fixed effects $(N=22)$. Among the different regression methods, we tested different linear, generalized, additive and mixed effects models (see Supplementary Material 3), and based on model performance, measured as the correlation between observed and fitted values, we selected generalized additive models (GAMs; R package 'mgcv'). GAMs are especially useful when the shape of the relationship between response and predictor variables is not known a priori (Guisan, Edwards, \& Hastie, 2002), and were performed using a Gaussian error distribution, an identity link function and three degrees of freedom for each factor that limit the level of smoothing to linear, quadratic and cubic responses (Wood, 2006).

Using multimodel inference (the 'MuMln' package in R; Barton, 2011) we explored all combinations of predictors and ranked models by their Akaike information criterion corrected for small sample size (AICC). Initially, we attempted to average results from models within 2 AICC units from the highest-ranked model (as in Early \& Sax, 2014; Li et al., 2014; Strubbe et al., 2015), but since only one model met this criterion for expansion (i.e., other models showed $\Delta \mathrm{AIC}>2$ ), we selected the highest-ranked model instead. The percentage of deviance explained was used to assess the goodness-of-fit of the highest-ranked model, and the deviance explained by each factor used in isolation was used to assess their relative importance. Mixed regression models (LME) including a random component to account for the taxonomic relatedness between species did not improve the model fit, but results from this option can be consulted in Supplementary Material 3.

Finally, we tested if a phylogenetic signal exists in the presence or absence of niche shifts in our data at the species level, which may be associated with traits that are dependent on evolutionary relatedness, although niche shifts, or a lack thereof, are probably not subject to natural selection. We estimated phylogenetic signal for niche unfilling and expansion using the Fritz and Purvis (2010) D statistic in R software using the 'caper' package (see Supplementary Material 4 for details).

\section{7 | Species distribution modelling}

To examine how levels of niche shift may relate to the predictive performance of ENMs, we constructed three sets of ENMs using the same occurrence and pseudo-absence data as for the niche analysis for each of the three geographical background extents. To do this we employed four different modelling algorithms within the 'BIOMOD2' package in 
R (Thuiller et al., 2014): generalized linear models (GLMs), generalized boosting regression (GBM), random forests (RF) and maximum entropy (MaxEnt). We allowed for variable selection and fitting to be done under default settings (as in Petitpierre et al., 2012; Strubbe et al., 2015), with all eight variables applied to each species. Prevalence weights for presence points and pseudo-absence points were set at neutral (0.5) (Mateo et al., 2015; Petitpierre et al., 2012). Models were trained on the native and native + invasive ranges for each species, with training data split for $70 \%$ training and $30 \%$ test data, and this was repeated 10 times for each modelling algorithm, giving a total of 40 model iterations per species, training extent and background. Model performance for each of the iterations was evaluated using area under the curve (AUC) and the true skill statistic (TSS). We built consensus predictions for each species by combining model outputs and keeping only models with good performance (AUC $>0.7$ and TSS $>0.5$ ) using the ensemble modelling functions of BIOMOD2. This final model output (single spatial prediction) was then assessed on how well it captured the invasive distribution by using the Boyce index. The Boyce index is a presence-only evaluation technique and ranges from -1 to 1 , and measures how much model predictions differ from a random distribution of observed presences across a prediction gradient (Hirzel, Le Lay, Helfer, Randin, \& Guisan, 2006): positive values indicate good model performance, values near 0 indicate models no better than random and negative values indicate prediction of poor suitability where there are presences (Broennimann et al., 2014). To examine any correlations between the Boyce index and niche change, we performed separate GLMs with the Boyce index as the dependent variable, and the percentage of overlap, unfilling or expansion, and background type as fixed effects in $\mathrm{R}$

\section{3 | RESULTS}

The final list of non-native invasive insect species included in this study is shown in Table 1. Our sensitivity tests revealed that the niche metric scores and ENM performance were influenced by the choice of geographical background (see Supplementary Material 2). To allow presentation of a single set of results, we selected for each species the geographical background that yielded the best transferability of ENMs as assessed through the Boyce index (Figures 1 and 3a). This was assessed on the models trained on native and invasive data and then projected to the invasive range, so that as many data as possible were incorporated in making this choice. The Boyce index and niche expansion and unfilling scores from all three backgrounds are included for comparison (Figure 2a, Supplementary Material 2). We found no evidence of phylogenetic signal or taxonomic bias in our approach (Supplementary Material 4).

\section{1 | Niche overlap}

Niche overlap in multivariate environmental space was low for all species (mean $D=0.21 \pm 0.11 S D$; Table 1). The null hypothesis for niche similarity (testing if the environmental niches occupied in both ranges are more similar than by chance) gave slightly different results depend- ing on the direction of the test (native compared with randomly generated invaded range, versus invaded compared with randomly generated native range) (Table 1). When comparing the actual native range with a random invaded range, niche similarity was rejected in nine cases. Niche similarity was also rejected for 11 of the species when comparing the actual invaded range with randomly generated samples across the native range (Table 1). For seven species niche similarity was rejected in both directions, and rejected for 14 unique species in at least one direction (Table 1).

When measuring niche change at the 75th percentile of density overlap between ranges, $54.5 \%$ (12) of the species exhibited niche expansion into new environments (Table 1, Figure 1). Likewise, niche unfilling was also highly variable, being displayed in $68.2 \%$ (15) of the species examined (Table 1, Figures 1 and 2a). While the niche expansion and unfilling scores remained generally consistent across different percentiles of density overlap, two species (Epiphyas postvittana and Anoplophora glabripennis) displayed niche expansion above $10 \%$ only when niche metrics were calculated above the 95th percentile (Supplementary Material 5).

\section{2 | Factors associated with niche change}

Regression models (GAM) explained $51 \%$ and $71 \%$ of the deviance in niche expansion and unfilling, respectively (Figure 2b,c, Table 2), which suggests that the geographical and human-related factors investigated here are relevant (albeit not the only) drivers of niche changes. Human influence in the native range was able to explain a considerable portion of the deviance in niche expansion (40\%), and also affected niche unfilling (34\%) (Figure 2b,c). It is worth noting that human influence in the native and invaded ranges was positively correlated (Pearson-moment correlation, $r=.60, p=.003$ ), suggesting that species tend to invade areas with a similar human influence to their native range. In addition, distance to roads in the invaded range was negatively associated with niche unfilling ( $54 \%$ of deviance explained), suggesting that roads act as important vectors of insect expansion (Fig. S3.3). Equatorial movement was shown to be positively associated with niche unfilling (28\%; Fig. S3.3), and negatively to niche expansion (4\%; Fig. S3.2). The size of the native range, poleward movement and the density of human population were not found to be correlated with either niche expansion or unfilling. We tried including relatedness between species (taxonomic order) either as a fixed or random factor in the models, but this option did not improve model fit. Furthermore, there was no statistically significant effect of phylogenetic signal on niche unfilling or expansion rates and it was not possible to differentiate the distribution of these niche metrics across the group from random or clumped patterns (see Supplementary Material 4).

\section{3 | Ecological niche model performance}

The overall ENM performance for these species was highly variable, ranging from very poor to good (Boyce index ranging from -0.73 to 0.85) for models trained on native range data only and fair to excellent for models trained on both native and invasive data (Boyce index 



H. destructor

L. decemlineata
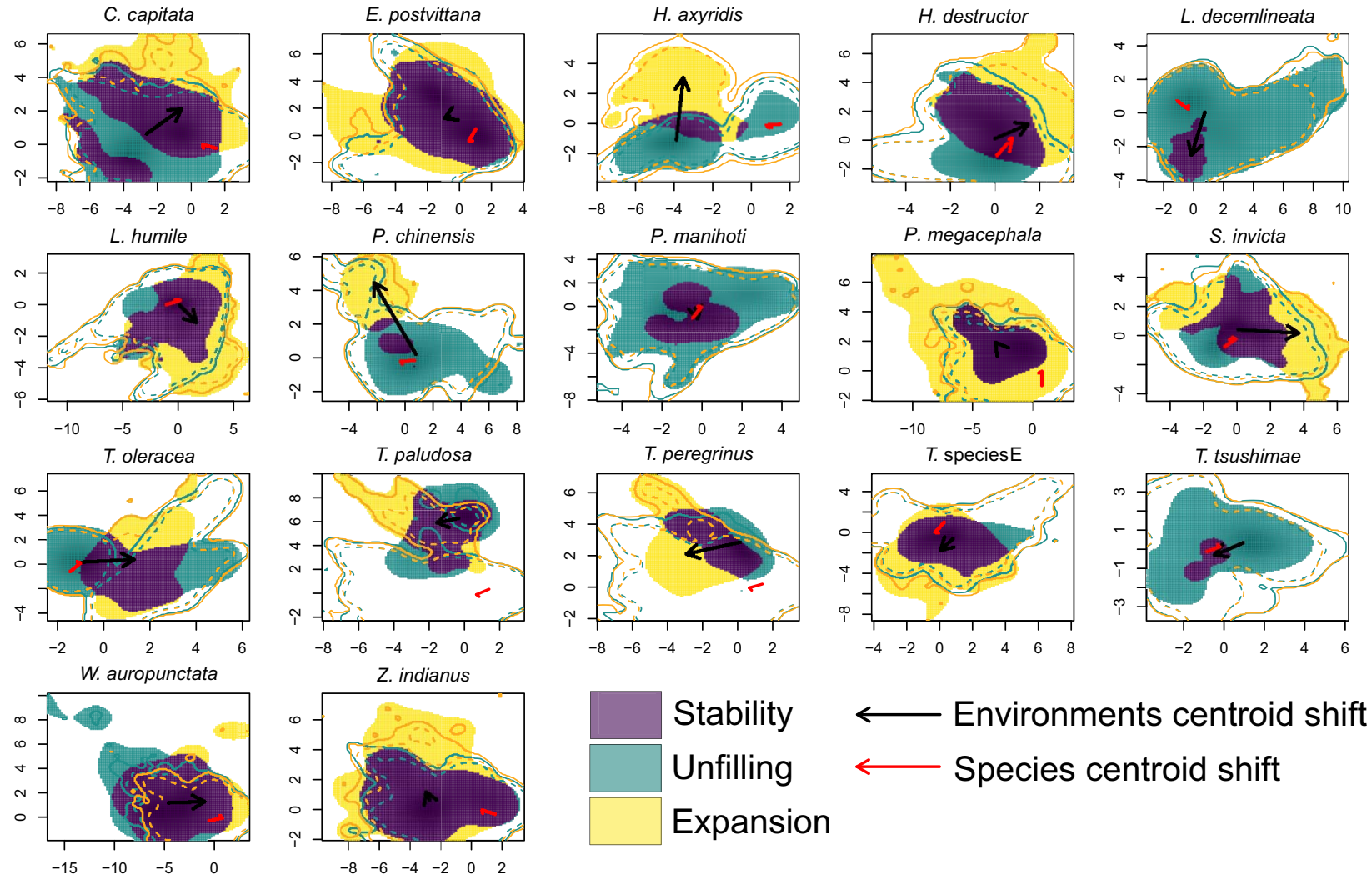

FIGURE 1 Climate niche surfaces overlaid between native and invasive ranges for 22 species of invasive insect. The pair of surfaces for each species was created on one of three background extents that gave the best environmental niche model transferability. The first two axes from the underlying principal components analysis are shown on the $x$ axis (PC1) and $y$ axis (PC2). Within each plot, the green area indicates niche unfilling, purple indicates niche stability and yellow indicates niche expansion. The solid lines indicate $100 \%$ of available climates for each respective background, the dashed line represents $75 \%$ of available climates. The red arrows indicate the change in the centre of the species niche between native and invasive ranges. The black arrows represent the shift in the centre of all environments going from native to invasive
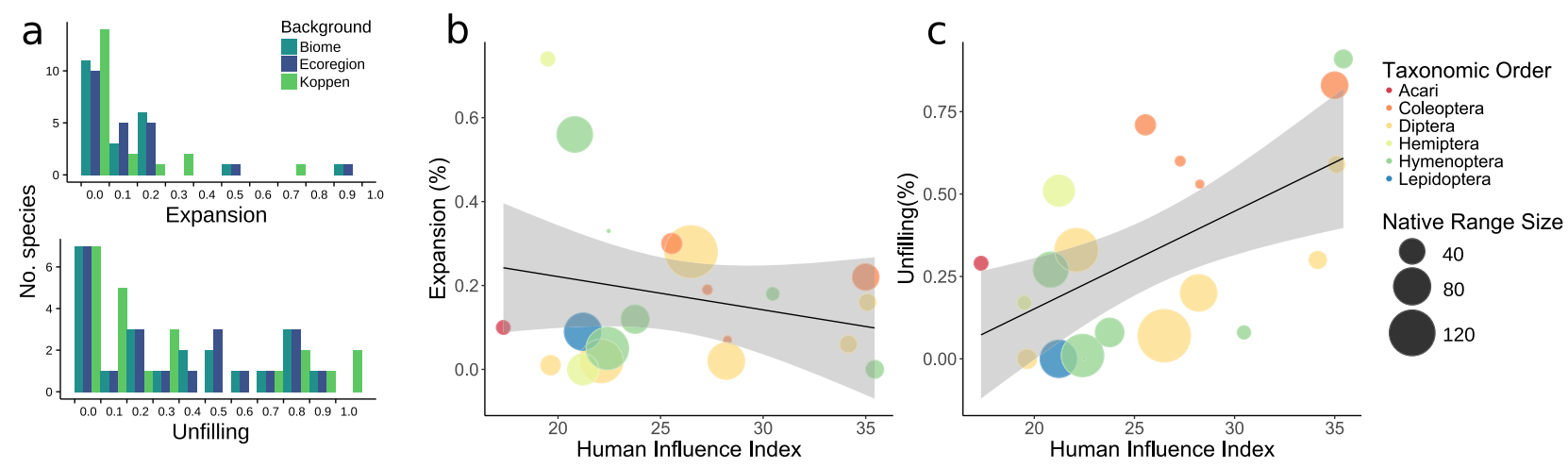

FIGURE 2 (a)Degree of niche expansion and unfilling shown in non-native invasive insects using three different geographical backgrounds: ecoregions, biomes and Köppen-Geiger climate classifications. (b), (c)Relationship between niche expansion and unfilling and the Human Influence Index of the native range. The bubble size is proportional to the size of the species native range (units are $\times 1,000 \mathrm{~km}^{2}$ ), and the colour represents the taxonomic order. Regression lines are calculated using generalized additive models that explained $40 \%$ and $34 \%$ of the variance in niche expansion and unfilling, respectively (see Supplementary Material 3). The shaded area represents $95 \%$ confidence intervals around the mean 
a

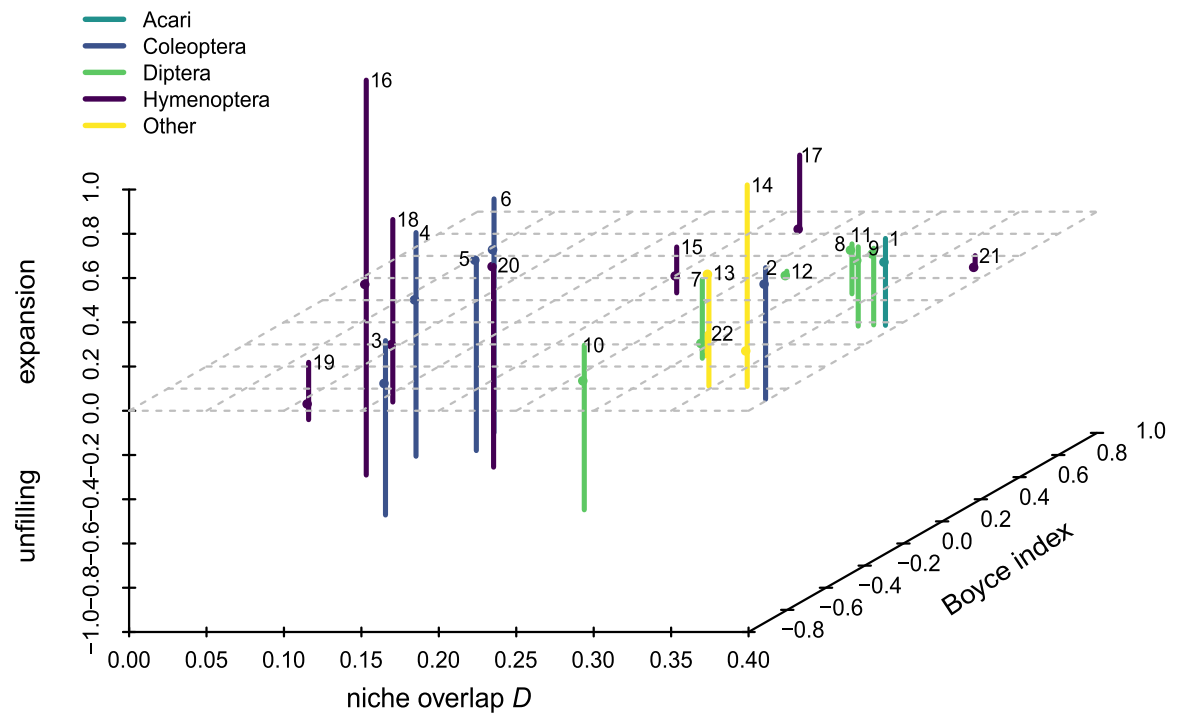

b
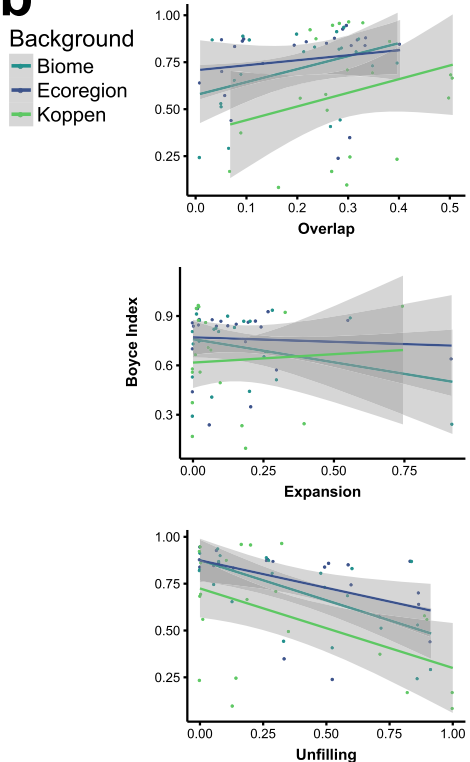

FIGURE 3 Niche changes, overlap and ecological niche model (ENM) performance between native and invaded ranges and for different geographical backgrounds for globally invasive insect species. (a) A 3-D scatterplot showing the niche change scores with ENM performance. The $y$ axis shows amounts of niche expansion and niche unfilling represented by the lines extending above and below the horizontal plane, respectively. Intersections with the zero plane are shown with filled dots. Niche overlap between native and invaded ranges in environmental space ( $D$ metric) is shown on the $x$ axis. The Boyce index on the $z$ axis shows the performance of species ENMs built on native range data and projected to the invaded or novel ranges. The Boyce index is a technique for evaluating ENMs that measures how model predictions differ from a random distribution of observed presences across a prediction gradient, and ranges from -1 (poor predictability) to 1 (good performance). Number labels correspond to species (Table 1). Values are reported in Table 1. (b)The three panels show the relationship between the Boyce index and the three different niche metrics using generalized linear models across the three different geographical backgrounds used in this study

values ranging from 0.41 to 0.97 ) (Table 1, Figure 3a). All Boyce index scores were calculated for the geographical background that yielded the best ENM performance (Table 1). All the modelling results are available in Supplementary Material 2. The Boyce index was negatively correlated with niche unfilling $\left(F_{1,62}=22.5, p<.001\right)$ and the backgrounds were significantly different $\left(F_{2,62}=5.432, p=.007\right)$ (Figure $\left.3 b\right)$. Niche overlap was not correlated with the Boyce index $\left(F_{1,62}=1.446\right.$,

TABLE 2 Results from generalized additive models using niche expansion and unfilling as dependent variables

\begin{tabular}{llll} 
Model & Adjusted $R^{2}$ & $\begin{array}{l}\text { Deviance } \\
\text { explained } \\
\text { by full Model }\end{array}$ & $\begin{array}{l}\text { Relative } \\
\text { deviance } \\
\text { explained by } \\
\text { predictors }\end{array}$ \\
\hline $\begin{array}{l}\text { Expansion } \\
\sim \text { HII + Equatorial }\end{array}$ & .40 & $51.2 \%$ & HII $39.8 \%$ \\
\hline $\begin{array}{l}\text { Unfilling } \sim \text { HII+ } \\
\text { Equatorial + Roads }\end{array}$ & .66 & $70.6 \%$ & Equatorial $3.9 \%$ \\
\hline & & & HII 33.5\% \\
\hline
\end{tabular}

The total deviance explained by the full model, and the deviance explained by each factor (when used individually) are provided. Due to redundancy (overlap) in the deviance explained by each factor individually, values do not necessarily add up to the deviance explained by the full model.

HII, human Influence in the native range; Equatorial, equatorial movement; Roads, distance to roads in the invaded range. $p=.234$ ); however, there was a significant difference between the backgrounds $\left(F_{2,62}=5.083, p=.009\right)$ (Figure 3b). Expansion did not display any significant association with the ENM performance $\left(F_{1,60}=0.255, p=.615\right)$ (Figure $\left.3 b\right)$.

\section{4 | DISCUSSION}

Both niche expansion and unfilling appear to be common in this assemblage of 22 globally invasive insects. Niche expansion was apparent in over half the species examined, indicating altered species-climate relationships during invasion. Using the same definitions of niche expansion and unfilling in previous multi-species studies (i.e. $>10 \%$ - Early and Sax, 2014; Petitpierre et al. 2012; Strubbe et al. 2013, 2015), these non-native invasive insects display rates of niche expansion higher than other taxonomic groups, with the exception perhaps of reptiles ( $\mathrm{Li}$ et al., 2014). The large amounts of niche unfilling exhibited here also suggest that many of the species investigated are not in climatic equilibrium and have the potential to further expand their invasive distributions because not all suitable climates are currently exploited. This is important because many non-native invasive insects will increasingly threaten human health, agricultural production and endemic biodiversity (Bradshaw et al., 2016).

Our results generally supported hypotheses of niche shift generated from previous work. Two species that demonstrated adaptive shifts in niche-limiting traits to facilitate range expansion, Aedes 
albopictus (Urbanski et al., 2012) and H. destructor (Hill et al., 2013), both displayed niche expansion here. The oriental fruit fly, Bactrocera dorsalis (Hill \& Terblanche, 2014) and the tortricid moth, E. postvittana (Lozier \& Mills, 2011), which both appeared to display high niche stability in recent attempts at ENM, had a high level of niche stability in our analyses. Likewise, recent ENM and niche shift analysis on the fire-ant Solenopsis invicta, concluded there had been niche expansion (Broennimann et al., 2012), which was also supported. We found that for some species where niche shift was concluded from previous ENM attempts, niche shift may be context dependent. The crane flies Tipula oleracea and Tipula paludosa demonstrated niche shift in previous work (Petersen, 2013). However, geographical background influenced the expansion score for both of these species (Supplementary Material 2), suggesting that background selection methods may give contradictory answers to whether a species demonstrates niche expansion. Previous work on traits for the electric ant W. auropunctata (Rey et al., 2012) and ENMs for the drosophilid Zaprionus indianus (da Mata et al., 2010) had concluded there was a niche shift, but we were unable to match these results across any geographical background or intersection of climates. For such cases, it may be that the definition of the native range was too broad and contained more types of occupied climates that lead to differences in niche shift metrics. Further, distinguishing niche shifts in shared multivariate climate space may also give more conservative results than ENM studies that include non-analogue climates.

Disturbance in the native habitat has been identified as a promoter of invasion success for some insects (Hufbauer et al., 2012), and here human influence was associated with both niche unfilling and expansion. This outcome mirrors results from invasive plant species that have been associated with disturbance in both native and introduced ranges, especially at the beginning of the invasion process (GonzálezMoreno et al., 2015). Further, non-native birds coming from areas in close contact with humans seem to be better pre-adapted to disturbed conditions and have a higher invasive potential than organisms coming from relatively pristine areas (Cardador, Carrete, Gallardo, \& Tella, 2016). Thus, new ranges under similar levels of human disturbance (e.g., agriculture and land clearing) to the native range, may facilitate invasions beyond expectations from climate alone. Niche unfilling has been associated with a species' 'opportunity to invade' (Cardador et al., 2016; Strubbe et al., 2013), and we found that distance from occurrence records to roads in the invaded range was associated with decreased niche unfilling in invasive insects. Transport networks, such as roads, facilitate the dispersal of invasive species by altering habitats, stressing native species and providing movement corridors along which they can disperse (Trombulak \& Frissell, 2000), and have been used to explain the spatial distribution of multiple invasive plants and animals (Gallardo et al., 2015).

Other factors, such as biotic interactions that may promote or impede insect invasions, or species life-history traits and rapid adaptation thereof, are likely to contribute to niche shifts. For example, natural habitats in the invaded range may offer stronger biotic resistance to the establishment of invaders than disturbed habitats (Gallardo \& Aldridge, 2013). The establishment of the Argentine ant, Linepithema humile, was previously found to be associated with suitable climate and high human pressure on the environment, with some small initial hindrance from native populations (Roura-Pascual et al., 2011). The relatively high incidence of niche shifts in insects may also be tied to their life-history traits (e.g., ectothermy, fecundity, phenology) and physiological tolerances, which have been associated with both establishment and invasion success (Jarošík, Kenis, Honěk, Skuhrovec, \& Pyšek, 2015). A number of species have displayed rapid adaptation in such traits during invasion (Hill et al., 2013; Gibert et al., 2016; Rey et al., 2012; Urbanski et al., 2012), suggesting that this could also be an important driver of observed niche shifts. It is important to note, however, that the species herein are already either serious economic pests or have a negative effect on biodiversity, hence limiting broader comment on invasions of non-native insect species that have different forms of impact (or minimal, impact). The fact that the species used in this study have reached this status may also be due to an inherent ability to change (or increased likelihood of changing) their species-climate relationships through niche shifts. Whilst adequate data for many nonnative insect species are sparse, investigation of niche change on an increased number of species across a broad range of ecosystems is required to further test the patterns we have observed.

Accurate predictions of insect invasions require that model selection, calibration and interpretation are backed by solid ecological understanding (Sutherst, 2014). ENMs built solely on the native range are often poor predictors of invasive ranges (Broennimann \& Guisan, 2008). We also found correlations between the predictive ability of an ENM and niche unfilling and overlap, supporting climate equilibrium as being important for model transferability. Given the high incidence of niche shifts and variability in model performance due to different contexts (background selection, variable selection and range definitions) for this group, it is apparent that predicting the distributions of nonnative invasive insects is not a 'one size fits all' approach (Sutherst, 2014). Modelling attempts should therefore explore these different contexts and associated sensitivity, especially for studies that aim to quantify invasion potential of important non-native species. In addition, some non-native invasive insect species are able to undergo rapid adaptation to novel environmental conditions (Gibert et al., 2016), and increasingly modified or cleared land for agricultural, forestry and urbanization purposes will also make predictions using static distribution datasets challenging. We suggest that ENMs should not be used in isolation to predict invasions, or invaded range extents, but need to be at least coupled with some analysis of changes in environmental/climatic space (and across a range of thresholds for marginal and nonanalogue climates) or, preferably, experiments aimed at identifying the underlying mechanisms (e.g., niche-limiting traits; see Hill et al., 2013; Rey et al., 2012.).

\section{ACKNOWLEDGMENTS}

The authors thank Petr Keil and two anonymous referees for constructive comments on this manuscript. The following people are thanked for supplying distribution data: Cleo Bertelsmeier, Mark De Meyer, Beat Wermelinger, Youhua Chen, James K. Wetterer, 
Matthew Petersen, Renata Alves da Mata, Olivier Rey, Li-Hsin Wu, Riaan Stals, Stefan Foord, Soroush Parsa, Florian Steiner and Birgit Schlick-Steiner. Diedrick Strubbe kindly shared R code for the 3D scatterplot in Figure 3. M.P.H. was partly supported through a National Research Foundation (NRF) of South Africa Knowledge Interchange and Collaboration (KIC) scholarship in relation to this project. B.G. was supported by a 'Juan de la Cierva' post-doctoral junior grant (JCL-2012-11908) by the Spanish Ministry of Economy and Competitiveness. J.S.T. is supported by the NRF Incentive Funding for Rated Researchers programme.

\section{DATA ACCESSIBILITY}

All species occurrence data used in this study are available at: https:// doi.org/10.6084/m9.figshare.4537922

\section{REFERENCES}

Anderson, R. P., \& Raza, A. (2010). The effect of the extent of the study region on GIS models of species geographic distributions and estimates of niche evolution: Preliminary tests with montane rodents (genus Nephelomys) in Venezuela. Journal of Biogeography, 37, 1378-1393.

Bradshaw, C. J. A., Leroy, B., Bellard, C., Roiz, D., Albert, C., Fournier, A. ... Courchamp, F. (2016). Massive yet grossly underestimated global costs of invasive insects. Nature Communications, 7, 12986.

Broennimann, O., Fitzpatrick, M. C., Pearman, P. B., Petitpierre, B., Pellissier, L., Yoccoz, N. G. ... Guisan, A. (2012). Measuring ecological niche overlap from occurrence and spatial environmental data. Global Ecology and Biogeography, 21, 481-497.

Broennimann, O., \& Guisan, A. (2008). Predicting current and future biological invasions: Both native and invaded ranges matter. Biology Letters, 4, 585-589.

Broennimann, O., Petitpierre, B., Randin, C., Engler, R., Di Cola, V., Breiner, F. ... Guisan, A. (2014). ecospat: Spatial ecology miscellaneous methods. R Package version 1.0 https://cran.r-project.org/web/packages/ecospat/index.html

Broennimann, O., Treier, U. A., Müller-Schärer, H., Thuiller, W., Peterson, A. T., \& Guisan, A. (2007). Evidence of climatic niche shift during biological invasion. Ecology Letters, 10, 701-709.

Busby, J. (1991). BIOCLIM - a bioclimate analysis and prediction system. In C. R. Margules \& M. P. Austin (Eds.), Nature conservation: Cost effective biological surveys and data analysis (pp. 64-68). Melbourne: CSIRO.

Cardador, L., Carrete, M., Gallardo, B., \& Tella, J.L. (2016). Combining trade data and niche modelling improves predictions of the origin and distribution of non-native European populations of a globally invasive species. Journal of Biogeography, 43, 967-978.

da Mata, R. A., Tidon, R., Côrtes, L. G., De Marco, P. Jr, \& Diniz-Filho, J. A. F. (2010). Invasive and flexible: niche shift in the drosophilid Zaprionus indianus (Insecta, Diptera). Biological Invasions, 12, 1231-1241.

De Meyer, M., Robertson, M. P., Mansell, M. W., Ekesi, S., Tsuruta, K., Mwaiko, W. ... Peterson, A. T. (2010). Ecological niche and potential geographic distribution of the invasive fruit fly Bactrocera invadens (Diptera, Tephritidae). Bulletin of Entomological Research, 100, 35-48.

Dray, S., \& Dufour, A. B. (2007). The ade4 package: Implementing the duality diagram for ecologists. Journal of Statistical Software, 22, 1-20.

Early, R., \& Sax, D. F. (2014). Climatic niche shifts between species' native and naturalized ranges raise concern for ecological forecasts during invasions and climate change. Global Ecology and Biogeography, 23, 1356-1365.

Fritz, S. A., \& Purvis, A. (2010). Selectivity in mammalian extinction risk and threat types: A new measure of phylogenetic signal strength in binary traits. Conservation Biology, 24, 1042-1051.

Gallardo, B., \& Aldridge, D. C. (2013). Priority setting for invasive species management: Integrated risk assessment of multiple Ponto Caspian invasive species into Great Britain. Ecological Applications, 23, 352-364.

Gallardo, B., Zieritz, A., \& Aldridge, D. C. (2015). The importance of the human footprint in shaping the global distribution of terrestrial, freshwater and marine invaders. PLoS One, 10, e0125801.

Gibert, P., Hill, M. P., Pascual, M., Plantamp, C., Terblanche, J. S., Yassin, A., \& Sgrò, C. M. (2016). Drosophila as models to understand the adaptive process during invasion. Biological Invasions, 18, 1089-1103.

González-Moreno, P., Diez, J. M., Richardson, D. M., \& Vilà, M. (2015). Beyond climate: Disturbance niche shifts in invasive species. Global Ecology and Biogeography, 24, 360-370.

Guisan, A., Edwards, T. C., \& Hastie, T. (2002). Generalized linear and generalized additive models in studies of species distributions: Setting the scene. Ecological Modelling, 157, 89-100.

Guisan, A., Petitpierre, B., Broennimann, O., Daehler, C., \& Kueffer, C. (2014). Unifying niche shift studies: Insights from biological invasions. Trends in Ecology and Evolution, 29, 260-269.

Harris, I. P. D. J., Jones, P. D., Osborn, T. J., \& Lister, D. H. (2014). Updated high-resolution grids of monthly climatic observations - the CRU TS3.10 Dataset. International Journal of Climatology, 34, 623-642.

Hill, M. P., Chown, S. L., \& Hoffmann, A. A. (2013). A predicted niche shift corresponds with increased thermal resistance in an invasive mite, Halotydeus destructor. Global Ecology and Biogeography, 22, 942-951.

Hill, M. P., Clusella-Trullas, S., Terblanche, J. S., \& Richardson, D.M. (2016). Drivers, impacts, mechanisms and adaptation in insect invasions. Biological Invasions, 18, 883-891.

Hill, M. P., \& Terblanche, J. S. (2014). Niche overlap of congeneric invaders supports a single-species hypothesis and provides insight into future invasion risk: Implications for global management of the Bactrocera dorsalis complex. PloS One, 9, e90121.

Hirzel, A. H., Le Lay, G., Helfer, V., Randin, C., \& Guisan, A. (2006). Evaluating the ability of habitat suitability models to predict species presences. Ecological Modelling, 199, 142-152.

Hufbauer, R. A., Facon, B., Ravigne, V., Turgeon, J., Foucaud, J., Lee, C. E. ... Estoup, A. (2012). Anthropogenically induced adaptation to invade (AIAI): Contemporary adaptation to human-altered habitats within the native range can promote invasions. Evolutionary Applications, 5, 89-101.

Jarošík, V., Kenis, M., Honěk, A., Skuhrovec, J., \& Pyšek, P. (2015). Invasive insects differ from non-invasive in their thermal requirements. PloS One, 10, e0131072.

Li, Y., Liu, X., Li, X., Petitpierre, B., \& Guisan, A. (2014). Residence time, expansion toward the equator in the invaded range and native range size matter to climatic niche shifts in non-native species. Global Ecology and Biogeography, 23, 1094-1104.

Lounibos, L. P. (2002). Invasions by insect vectors of human disease. Annual Review of Entomology, 47, 233-266.

Lozier, J. D., \& Mills, N. J. (2011). Predicting the potential invasive range of light brown apple moth (Epiphyas postvittana) using biologically informed and correlative species distribution models. Biological Invasions, 13, 2409-2421.

Mateo, R. G., Broennimann, O., Petitpierre, B., Muñoz, J., van Rooy, J., Laenen, B. ... Vanderpoorten, A. (2015). What is the potential of spread in invasive bryophytes? Ecography, 38, 480-487. 
Olson, D. M., Dinerstein, E., Wikramanayake, E. D., Burgess, N. D., Powell, G. V. N., Underwood, E. C. .. Kassem, K. R. (2001). Terrestrial ecoregions of the world: A new map of life on earth. BioScience, 51, 933-938.

Parravicini, V., Azzurro, E., Kulbicki, M., \& Belmaker, J. (2015). Niche shift can impair the ability to predict invasion risk in the marine realm: An illustration using Mediterranean fish invaders. Ecology Letters, 18, 246-253.

Petersen, M. J. (2013). Evidence of a climatic niche shift following North American introductions of two crane flies (Diptera; genus Tipula). Biological Invasions, 15, 885-897.

Petitpierre, B., Kueffer, C., Broennimann, O., Randin, C., Daehler, C., \& Guisan, A. (2012). Climatic niche shifts are rare among terrestrial plant invaders. Science, 335, 1344-1348.

Pyšek, P., Jarošík, V., Hulme, P. E., Kühn, I., Wild, J., Arianoutsou, M. . . Winter, M. (2010). Disentangling the role of environmental and human pressures on biological invasions across Europe. Proceedings of the National Academy of Sciences, 107, 12157-12162.

Rey, O., Estoup, A., Vonshak, M., Loiseau, A., Blanchet, S., Calcaterra, L. ... Facon, B. (2012). Where do adaptive shifts occur during invasion? A multidisciplinary approach to unravelling cold adaptation in a tropical ant species invading the Mediterranean area. Ecology Letters, 15, 1266-1275.

Rödder, D., \& Lötters, S. (2009). Niche shift versus niche conservatism? Climatic characteristics of the native and invasive ranges of the Mediterranean house gecko (Hemidactylus turcicus). Global Ecology and Biogeography, 18, 674-687.

Roura-Pascual, N., Hui, C., Ikeda, T., Leday, G., Richardson, D. M., Carpintero, S. ... Krushelnycky, P. (2011). Relative roles of climatic suitability and anthropogenic influence in determining the pattern of spread in a global invader. Proceedings of the National Academy of Sciences USA, 108, 220-225.

Rubel, F., \& Kottek, M. (2010). Observed and projected climate shifts 1901-2100 depicted by world maps of the Köppen-Geiger climate classification. Meteorologische Zeitschrift, 19, 135-141.

Sanderson, E. W., Jaiteh, M., Levy, M. A., Redford, K. H., Wannebo, A. V., \& Woolmer, G. (2002). The Human Footprint and the Last of the Wild. The human footprint is a global map of human influence on the land surface, which suggests that human beings are stewards of nature, whether we like it or not. Bioscience, 52, 891-904.

Strubbe, D., Beauchard, O., \& Matthysen, E. (2015). Niche conservatism among non-native vertebrates in Europe and North America. Ecography, 38, 321-329.

Strubbe, D., Broennimann, O., Chiron, F., \& Matthysen, E. (2013). Niche conservatism in non-native birds in Europe: Niche unfilling rather than niche expansion. Global Ecology and Biogeography, 22, 962-970.

Sutherst, R. W. (2014). Pest species distribution modelling: Origins and lessons from history. Biological Invasions, 16, 239-256.

Thuiller, W., Georges, D., \& Engler, R. (2014). biomod2: Ensemble platform for species distribution modeling. R package version 3.3-7. http:// CRAN.R-project.org/package $=$ biomod 2 .
Trombulak, S. C., \& Frissell, C. A. (2000). Review of ecological effects of roads on terrestrial and aquatic communities. Conservation Biology, 14, 18-30.

Urbanski, J., Mogi, M., O’Donnell, D., DeCotiis, M., Toma, T., \& Armbruster, P. (2012). Rapid adaptive evolution of photoperiodic response during invasion and range expansion across a climatic gradient. The American Naturalist, 179, 490-500.

Vitousek, P. M., D’Antonio, C. M., Loope, L. L., \& Westbrooks, R. (1996). Biological invasions as global environmental change. American Scientist, 84, 468-478.

Warren, D. L., Glor, R. E., \& Turelli, M. (2008). Environmental niche equivalency versus conservatism: Quantitative approaches to niche evolution. Evolution, 62, 2868-2883.

Wiens, J. J., \& Graham, C. H. (2005). Niche conservatism: Integrating evolution, ecology, and conservation biology. Annual Review of Ecology, Evolution, and Systematics, 36, 519-539.

Wiens, J. A., Stralberg, D., Jongsomjit, D., Howell, C. A., \& Snyder, M. A. (2009). Niches, models, and climate change: Assessing the assumptions and uncertainties. Proceedings of the National Academy of Sciences USA, 106, 19729-19736.

Wood, S. N. (2006). Generalized additive models: An introduction with R. Boca Raton, FL: Chapman \& Hall/CRC.

\section{BIOSKETCHES}

Matт Hill was a post-doctoral fellow at the Centre for Invasion Biology, Stellenbosch University. His research interests cover macroecology, biogeography and invasion ecology of agriculturally important terrestrial invertebrates.

BeLINDA Gallardo is a post-doctoral researcher at the Pyrenean Institute of Ecology (Spanish National Research Council, CSIC). She is specialized in the risk assessment of invasive species, with particular interest in (but not restricted to) the aquatic environment.

John Terblanche is a professor at Stellenbosch University with research interests in invasion biology, physiological ecology and evolutionary biology.

\section{SUPPORTING INFORMATION}

Additional Supporting Information may be found online in the supporting information tab for this article.

How to cite this article: Hill MP, Gallardo B, Terblanche JS. A global assessment of climatic niche shifts and human influence in insect invasions. Global Ecol Biogeogr. 2017;26:679-689. https://doi.org/10.1111/geb.12578 